\title{
SCLEROSTIN REGULATION, MICROARCHITECTURE, AND ADVANCED \\ GLYCATION END-PRODUCTS IN THE BONE OF ELDERLY WOMEN WITH TYPE 2 \\ DIABETES
}

Alessandra Piccoli, $\mathrm{PhD}^{1,2}$; Francesca Cannata, MS¹; Rocky Strollo, MD, PhD'; Claudio Pedone, MD³; Giulia Leanza, PhD; Fabrizio Russo, MD; Valentina Greto, PhD; Camilla Isgrò, MS5; Carlo Cosimo Quattrocchi, MD, $\mathrm{PhD}^{6}$; Carlo Massaroni, $\mathrm{PhD}^{7}$; Sergio Silvestri, PhD ${ }^{7}$; Gianluca Vadalà, MD, $\mathrm{PhD}^{4}$; Tiziana Bisogno, $\mathrm{PhD}^{8}$; Vincenzo Denaro, MD; Paolo Pozzilli, MD¹, Simon J. Tang, $\mathrm{PhD}^{9}$; Matt J. Silva, $\mathrm{PhD}^{9}$; Caterina Conte, MD, $\mathrm{PhD}^{10,11}$, Rocco Papalia, MD, PhD4; Mauro Maccarrone, MS, PhD²,12; Nicola Napoli, MD, PhD ${ }^{1,13}$

${ }^{1}$ Unit of Endocrinology and Diabetes, Departmental Faculty of Medicine and Surgery, Campus Bio-Medico University of Rome, Italy

${ }^{2}$ Unit of Biochemistry and Molecular Biology, Departmental Faculty of Medicine and Surgery, Campus Bio-Medico University of Rome, Italy

${ }^{3}$ Unit of Geriatrics, Departmental Faculty of Medicine and Surgery, Campus Bio-Medico university of Rome, Italy

${ }^{4}$ Unit of Orthopedic and Trauma Surgery, Departmental Faculty of Medicine and Surgery,

Campus Bio-Medico University of Rome, Italy

${ }^{5}$ Department of Basic Medical Sciences, Neurosciences and Sensory Organs, University of Bari "Aldo Moro", Bari, Italy

${ }^{6}$ Department of Medicine, Unit of Radiology, Campus Bio-Medico University of Rome, Italy

This article has been accepted for publication and undergone full peer review but has not been through the copyediting, typesetting, pagination and proofreading process which may lead to differences between this version and the Version of Record. Please cite this article as doi: $10.1002 / \mathrm{jbmr} .4153$ 
${ }^{7}$ Research Unit of Measurements and Biomedical Instrumentation, Departmental Faculty of Bioengineering, Campus Bio-Medico di Roma University, Italy ${ }^{8}$ Endocannabinoid Research Group, Institute of Translational Pharmacology, CNR, Italy ${ }^{9}$ Unit of Orthopedics, Washington University in Saint Louis, USA

${ }^{10}$ Division of Immunology, Transplantation and Infectious Diseases, IRCCS Ospedale San Raffaele, Milan, Italy ORCID 0000-0001-7066-5292

${ }^{11}$ Vita-Salute San Raffaele University, Milan, Italy

${ }^{12}$ European Center for Brain Research (CERC)/Santa Lucia Foundation, Rome, Italy ${ }^{13}$ Division of Bone and Mineral Diseases, Washington University in ST Louis, St Louis MO, USA

Running title: Bone fragility in T2D women

\section{Corresponding authors:}

Nicola Napoli, MD, PhD; Unit of Endocrinology and Diabetes, Campus Bio-Medico University of Rome, Via Alvaro del Portillo 21, 00121, Rome, Italy; n.napoli@unicampus.it; URCID: 0000-0002-3091-8205 


\section{Disclosures}

All authors have nothing to disclose relevant to this work. 


\section{Abstract.}

Increased circulating sclerostin and accumulation of advanced glycation end-products (AGEs) are two potential mechanisms underlying low bone turnover and increased fracture risk in type 2 diabetes (T2D). Whether the expression of the sclerostin-encoding SOST gene is altered in T2D, and whether it is associated with AGEs accumulation or regulation of other bone formation-related genes is unknown. We hypothesized that AGEs accumulate and SOST gene expression is upregulated in bones from subjects with T2D, leading to downregulation of bone forming genes (RUNX2 and osteocalcin) and impaired bone microarchitecture and strength. We obtained bone tissue from femoral heads of 19 T2D postmenopausal women (mean HbA1c 6.5\%) and 73 age- and BMI-comparable nondiabetic women undergoing hip replacement surgery. Despite similar bone mineral density (BMD) and biomechanical properties, we found a significantly higher SOST $(p=0.006)$ and a parallel lower RUNX2 ( $p=0.025)$ expression in T2D compared with non-diabetic subjects. Osteocalcin gene expression did not differ between T2D and non-diabetic subjects, as well as circulating osteocalcin and sclerostin levels. We found a 1.5-fold increase in total bone AGEs content in T2D compared with non-diabetic women (364.8 \pm 78.2 vs. $209.9 \pm 34.4 \mu \mathrm{g}$ yuinine/g collagen, respectively; $p<0.001$ ). AGEs bone content correlated with worse bone microarchitecture, including lower volumetric BMD ( $r=-0.633 ; p=0.02)$, BV/TV ( $r=-0.59$; $p=0.033)$ and increased trabecular separation/spacing $(r=0.624 ; p=0.023)$. In conclusion, our data show that even in patients with good glycemic control, T2D affects the expression of genes controlling bone formation (SOST and RUNX2). We also found that accumulation 
of AGEs is associated with impaired bone microarchitecture. We provide novel insights that may help understand the mechanisms underlying bone fragility in T2D.

Key words: Diabetes, Sclerostin, Osteoblasts, Bone $\mu \mathrm{CT}$, Biomechanics 


\section{Introduction}

Diabetes mellitus is one of the most common chronic diseases worldwide, whose prevalence has increased in parallel with the increase in obesity and changes in lifestyle. ${ }^{(1)}$ Fragility fractures are important complications of both type 1 and type 2 diabetes (T1D and T2D, respectively), independent of age and sex, suggesting a strong involvement of glucose metabolism in bone homeostasis. ${ }^{(2)}$ Several epidemiological studies highlighted an increased fracture risk among T2D subjects, despite normal or even higher BMD as compared with healthy controls. ${ }^{(3,4)}$ This suggests abnormalities in bone quality, with bone material strength and/or bone microarchitecture likely being affected. ${ }^{(5,6)}$ Alterations in bone turnover and bone cell function may contribute to impaired bone quality in diabetes. Several evidences highlighted that circulating biochemical markers of both bone formation (i.e. osteocalcin, procollagen type I N-terminal propeptide [PINP] and bone-specific alkaline phosphatase) and bone resorption such as C-terminal telopeptide of type 1 collagen (CTX) are decreased in T2D subjects, indicating that diabetes is characterized by low bone turnover. (4,7-9) $^{-1}$

silerostin, a glycoprotein encoded by SOST and secreted by osteocytes, is a potent inhibitor of the canonical WNT signaling pathway and therefore a negative regulator of bone formation. Sclerostin expression progressively increases during osteocyte maturation, and higher levels were reported in osteocytes surrounded by mineralized bone. ${ }^{(10)}$ We have shown that serum sclerostin is increased in T2D (2), and tends to correlate with glucose control (glycated hemoglobin, $[\mathrm{HbA} 1 \mathrm{c}]$ ) in T2D and higher vertebral marrow fat. ${ }^{(8,11)}$ 
Moreover, some studies described a positive association between serum sclerostin and vertebral fractures in postmenopausal women with T2D compared to healthy subjects, suggesting that sclerostin plays a role in diabetes-associated bone fragility. ${ }^{(12)}$ However, it is unknown if SOST gene expression is upregulated in T2D and whether other bone formation-related genes are affected.

Accumulation of advanced glycation end-products (AGEs), which are markedly increased in subjects with diabetes, may further contribute to reduced bone formation and impaired bone quality. (13) Previous studies demonstrated that AGEs accumulate in diabetes, forming nonenzymatic cross-links within type I collagen (which constitutes nearly $90 \%$ of bone organic phase), affecting biochemical and mechanical properties of bone. ${ }^{(14)}$

Although many hypotheses have been proposed regarding the mechanisms underlying impaired bone quality and/or its mechanical properties in hyperglycemic states, how T2D contributes to fracture risk has not been fully elucidated. We sought to assess the relationship between bone microarchitecture and strength with bone AGEs content and the expression of SOST and bone forming genes (RUNX2 and osteocalcin) at the tissue level. To this aim, we evaluated the gene expression of both bone formation and WNT pathway markers in bone samples from postmenopausal women with or without T2D undergoing hip replacement surgery, and examined the association between diabetes and bone density, geometry and strength in this population.

\section{Materials and Methods}




\section{Study subjects}

Between 2015 and 2017, postmenopausal women affected by osteoarthritis who were scheduled for elective hip replacement surgery were consecutively screened for participation in this cross-sectional study. All participants provided written informed consent.

The study protocol was reviewed and approved by the Ethics Committee of Campus BioMedico University of Rome. Diabetes diagnosis was confirmed by the treating diabetes physician and based on the 2015 American Diabetes Association criteria as follows: "fasting plasma glucose $(F P G) \geq 126 \mathrm{mg} / \mathrm{dl}$ or a 2 -h plasma glucose $(2-\mathrm{h} P G) \geq 200 \mathrm{mg} / \mathrm{dl}$ during a 75 -g oral glucose tolerance test (OGTT), or a hemoglobin A1c $(\mathrm{HbA} 1 \mathrm{c}) \geq 6.5 \%$. In the absence of unequivocal hyperglycemia, diagnosis required two abnormal test results from the same sample or in two separate test samples".(15) Exclusion criteria were as follows: age $<65$ years, use of medications affecting bone metabolism such as estrogen, raloxifene, tamoxifen, bisphosphonates, teriparatide, denosumab, thiazolidinediones, glucocorticoids, anabolic steroids and phenytoin. Further exclusion criteria were: evidence of hypercalcemia, hypocalcemia, chronic liver disease, renal failure, hypercortisolism, malabsorption and immobilization, current alcohol or tobacco use, evidence of bone metastases or disease at the site of surgery.

Prior to surgery, participants underwent a complete physical examination and dual energy X-ray absorptiometry (DXA).

\section{Sample preparation}


Blood samples were collected in the morning after an overnight fast, before surgery, and stored at $-80^{\circ} \mathrm{C}$ until use.

Discarded femoral head specimens were collected fresh and stored without any fixative at $4{ }^{\circ} \mathrm{C}$ until procession by our study team. RNA extraction was performed from bone tissue within $24 \mathrm{~h}$ after surgery. A trabecular bone specimen was sampled from the femoral head (approximately $1 \times 1.5 \mathrm{~cm}$ ) and transferred to a $50 \mathrm{ml}$ tube containing sterile PBS $1 \mathrm{X}$. Samples were repeatedly flushed in order to remove the marrow, vigorously vortexed and the supernatant was discharged. We repeated the washing process at least twice and finally bone samples were snap frozen and stored at $-80 \mathrm{C}^{\circ}$. Moreover, a trabecular core $(10 \mathrm{~mm}$ diameter $\times 20 \mathrm{~mm}$ length) was excised from femoral head alongside the direction of the principal trabeculae ${ }^{(16)}$ for compression testing. Compression tests were performed using a fresh trabecular core, without freezing and thawing. Femoral head samples were rapidly stored by our study team at $-80^{\circ} \mathrm{C}$ until use.

\section{Isolation of mRNA and evaluation of gene expression with RT-PCR}

Total RNA was isolated using TRIzol Reagent (Invitrogen Corp., Carlsbad, CA), ${ }^{(17)}$ following manufacturer's instructions, and RNA concentration was determined spectrophotometrically (TECAN, InfiniteM200PRO). The ratio of absorbance at 260 and $280 \mathrm{~nm}\left(\mathrm{~A}_{260 / 280}\right)$ was used to assess the purity of isolated RNA. Bone tissue RNA yields ranged between 1.8 and 2 . We only reverse transcribed RNA within this range, excluding from the analysis those samples not reaching this threshold. In order to carry out the quantitative real-time RT-PCR, $1 \mu \mathrm{g}$ of total RNA was reverse transcribed using High-Capacity cDNA Reverse Transcription 
Kit (Applied Biosystems, Carlsbad, CA), according to product protocol $\left(25^{\circ} \mathrm{C}\right.$ for 10 minutes, $37^{\circ} \mathrm{C}$ for 2 hours, $85^{\circ} \mathrm{C}$ for 5 minutes). A further real time RT-PCR analysis was performed using TaqMan Gene Expression Analysis Assay, with the 7900 Real-Time PCR system (Applied Biosystems, Carlsbad, CA) and standard cycling conditions $\left(95^{\circ} \mathrm{C}\right.$ for 10 minutes; 40 cycles of $95^{\circ} \mathrm{C}$ for 15 seconds and $60^{\circ} \mathrm{C}$ for 1 minute; followed by $95^{\circ} \mathrm{C}$ for 15 seconds, $60^{\circ} \mathrm{C}$ for 15 seconds and $95^{\circ} \mathrm{C}$ for 15 seconds). Gene expression of sclerostin (SOST, Taqman ID Hs00228830_m1), bone formation marker osteocalcin (OCN, Taqman ID Hs01587814_g1) and Runt related transcription factor 2 (RUNX2, Taqman ID Hs01047973_m1) was evaluated in bone tissue biopsies. For each analysis, we used three internal controls (housekeeping genes), the most stable ones. Gene expression was normalized with ribosomal RNA18S (Hs03928985_g1), GAPDH (Hs02758991_g1) and BACTIN (Hs01060665_g1), and the results were analyzed using BestKeeper software tool.(1820)

\section{Bone density (DXA) and micro-computed tomography (mCT)}

Lumbar spine (L1 to L4), total and neck femoral BMD were measured by DXA (QDR- 4500A, Hologic, Inc., Bedford Massachusetts) at lumbar spine and proximal femur. The coefficient of variation $(\mathrm{CV})$ for this technique at our center was $1.1 \%$ for the lumbar spine and $1.2 \%$ for the proximal femur. Standardized procedures and scan analysis were executed for all scans. ${ }^{(21)}$

Bone cores (8- $\mathrm{mm}$ in diameter and 8-10 $\mathrm{mm}$ in length) were harvested from the femoral head of each donor and then scanned using a benchtop microCT system (microCT 40; 
Scanco Medical AG, Bruttisellen, Switzerland). Voxels of $15 \times 15 \times 15 \mu m^{3}$ in dimension were obtained with an X-ray tube potential of $70 \mathrm{kVp}$, intensity of $114 \mathrm{~mA}, 1000$ projections, and $300 \mathrm{~ms}$ integration time. To standardize the procedure, cores were obtained as close as possible to the principal trabecular loading axis of the femoral head as identified from the digital X-ray. A hydroxyapatite phantom calibration with the manufacturer's specifications was performed weekly for this system. The trabecular bone analyses were conducted such that contours excluded the cortical bone. After contouring, trabecular bones were masked by lower and upper Thresholds that partitioned a digital image into the boney regions.

Tissue mineral density (TMD) was defined as the mean of volumetric bone mineral density for all voxels assigned to the matrix (voxels with a BMD more than $600 \mathrm{mg} \mathrm{HA} / \mathrm{cm}^{3}$ ). Standard trabecular architecture parameters were also computed including the bone volume fraction (BV/TV), connectivity density (Conn. D), trabecular number (Tb.N), trabecular spacing (Tb.Sp), and trabecular thickness (Tb. Th). Analyses were completed using by Scanco Evaluation program.

\section{Biomechanical tests}

A cylindrical specimen with a diameter of $10 \mathrm{~mm}$ and a length of $20 \mathrm{~mm}$ was obtained from each femoral head sample and biomechanical tests were performed on fresh trabecular cores. An electromechanical testing machine (Mod. 3365, Instron Corp., Canton, MA, USA) was used to perform the mechanical compression tests. Each cylindrical specimen was positioned between two compression platens onto the testing machine ensuring the alignment of the specimen with the machine axis. The impressed force was measured by a 
static load force cell (Mod. 2530-447, Instron Corp., Canton, MA, USA) with a force capacity of $\pm 5 \mathrm{kN}$, linearity $\pm 0.25 \%$ of reading and repeatability of $\pm 0.25 \%$ of reading. The specimen strain during the test was obtained by recording the crosspiece movement, with an accuracy of $\pm 0.02 \mathrm{~mm} 25$. Bluehill software (Instron Corp., Canton, MA, USA) was used for controlling the compression tests, and to record the force and deformation data. The strain rate was set to $0.01 \mathrm{~s}-1$. Data were recorded at $25 \mathrm{~Hz}$. Young's modulus, ultimate strength and yield strength of each specimen were obtained from the specimen strain-stress curve in MATLAB (MathWorks, version R2017b). Young's modulus was obtained as the slope of the linear part of the calculated stress-strain curve. ${ }^{(22)}$ We used the Ultimate strength to calculate the max load and stress required to initiate the plastic deformation of bone, while the Yield strength as the minimum load or stress required to maintain the plastic behavior of bone. ${ }^{(23)}$ 


\section{Measurement of advanced glycation end-products (AGEs) in bone}

Pieces of trabecular bone (approximately $4 \times 4 \times 4 \mathrm{~mm}^{3}$ in volume) were isolated from the surgical specimens of a subset of 5 diabetics and 15 non-diabetics. The samples were first demineralized using ethylenediaminetetra acetic acid, enzymatically homogenized using papain $\left(5 \mu \mathrm{g} / \mathrm{mL}, 65^{\circ} \mathrm{C}\right.$ for 18 hours), and then finally hydrolyzed in $12 \mathrm{~N}$ hydrochloric acid $\left(12 \mathrm{~N} \mathrm{HCl}, 120^{\circ} \mathrm{C}\right.$ for $\left.3 \mathrm{~h}\right)$. The samples were then centrifuged, and the supernatant collected and then plated in triplicate with quinine sulfate standards. Fluorescence emission was read at a wavelength of $440 \mathrm{~nm}$ with an excitation wavelength of $370 \mathrm{~nm}$ using a micro-plate photometer (SpectraMax M2, Molecular Devices, Sunnyvale, CA). The AGEs content was then normalized to the amount of collagen present in each sample, which was quantified using a chloramine T colorimetric assay and standardized to hydroxyproline.(24,25)

\section{Biochemical analyses}

Fasting morning blood samples were analyzed by standard methods for the evaluation of glucose, calcium, creatinine, serum urea. Serum concentration of sclerostin was performed with enzyme immunoassay (Biomedica $\mathrm{GmbH}$, Vienna, Austria). The determination of serum undercarboxylated osteocalcin (Glu-OCN) and of carboxylated fraction (Gla-OCN) was carried out through commercially available standard immunoassay (Takara Bio Inc., Nojihigashi, Kusatsu, Shiga, Japan), according to manufacturer's protocol. 


\section{Statistical analyses.}

Data were analyzed using R 3.6.1 (R Foundation for Statistical Computing, Vienna, Austria). Patients' characteristics were described using means and standard deviations or medians and interquartile ranges, as appropriate, and percentages. Student's t-test or the Wilcoxon rank-sum test were used as appropriate to compare variables between groups. When variables were not normally distributed, we used a logarithmic transformation. Pearson's correlation coefficients were used to assess the relationship between variables.

\section{Results}

\section{Subject characteristics}

A total of 92 postmenopausal women scheduled for elective hip replacement surgery were enrolled (19 T2D subjects and 73 non-diabetic controls). Demographic and clinical characteristics of subjects are presented in Table 1. Non-diabetic and T2D subjects had similar age, BMI and menopausal age (Table 1). Mean disease duration in diabetic subjects was 14.6 years (SD 9.8 years). HbA1c was collected in all T2D subjects within three months before surgery and in average was 6.5\% SD1.7 (Table 1). Diabetes medications used by T2D subjects included monotherapy with metformin $(n=11)$ and combination therapy with metformin plus insulin and glinide $(n=5)$ or glinide $(n=3)$.

As expected, T2D subjects showed significantly higher fasting blood glucose compared to controls (120.1 mg/dl, SD 21.5 vs. 100.7 mg/dl, SD 11.21, respectively, $p=0.002)$ (Table 1). 


\section{Bone Gene expression and serum markers}

We analyzed gene expression at bone level, of the WNT pathway inhibitor SOST, the bone forming transcriptional factor $R U N X 2$, and the bone matrix protein $O C N$. Then, circulating levels of sclerostin and OCN (Gla-OCN and Glu-OCN) were also measured. Gene expression results, expressed in logarithmic scale, are shown in Figure 1. SOST gene expression in the bone of 53 analyzed subjects (15 T2D subjects and 38 non-diabetic) revealed significantly higher mRNA levels in T2D subjects vs. non-diabetics $(p=0.006$, Figure 1.A), even independently of age and BMI ( $p=0.007)$.

When we analyzed bone gene expression of RUNX2 and OCN in a total number of 39 enrolled subjects (15 T2D subjects and 24 non-diabetic controls), we found that RUNX2 mRNA levels were lower in T2D subjects versus controls ( $p=0.025$, Figure 1.B).

Finally, OCN gene expression did not differ between T2D subjects and non-diabetics, $(p=0.721$, Figure 1.C).

Circulating levels of both Gla-OCN and Glu-OCN were not different between T2D subjects and non-diabetics. Gla-OCN was 14.1 (SD 5.2) ng/ml in non-diabetic vs. 14.9 (SD 6.0) ng/ml „I. T2D subjects, while Glu-OCN was 3.3 (SD 2.9) ng/ml in non-diabetic vs. 2.96 (SD 1.79) $\mathrm{ng} / \mathrm{ml}$ in T2D subjects ( $p=0.88)$. Sclerostin was higher in T2D subjects, but this difference was not significant (28.5, SD $3.2 \mathrm{pmol} / \mathrm{L}$ vs. 22.8, SD $1.4 \mathrm{pmol} / \mathrm{L} ; \mathrm{p}=0.25)$. 


\section{Bone density and bone microarchitecture}

Based on femoral BMD by DXA interpreted according to the World Health Organization (WHO) criteria, ${ }^{(26)} 32.1 \%$ of non-diabetic and $50 \%$ of T2D subjects were osteopenic (T-score between -1.0 SD and -2.5 SD), while 3.6\% and 0\% were osteoporotic (T-score less than 2.5 SD), respectively. Comparison of BMD at lumbar spine and hip BMD (neck and total) showed no significant differences among T2D and non-diabetic subjects (Table 2).

According to microarchitecture assessment by $\mathrm{MCT}$ on bone core samples, T2D and nondiabetic subjects had similar trabecular indices, including bone volume density (BV/TV), connectivity, mean trabecular thickness, trabecular number, trabecular separation, tissue mineral density and volumetric BMD.

\section{AGEs and biomechanical tests in bone sample cores.}

We found a significant 1.5 -fold increase in total fluorescent AGEs in T2D subjects $(n=5)$

nnmpared to non-diabetic ones $(n=15)(364.8 \pm 78.2 \mu$ g quinine/g collagen vs. $209.9 \pm 34.4$ $\mu g$ quinine/g collagen, $p<0.001$ ) (Figure 2).

Correlation analysis, including both T2D and non-diabetic subjects, showed that AGEs were negatively correlated with volumetric BMD, BV/TV and positively correlated with trabecular separation and spacing (Figure 3, panels A, B, C). 
Young's modulus, upper yield strength and lower yield strength values were compared between T2D and non-diabetic subjects. Results were not different between T2D and nondiabetic subjects (Table 2).

\section{Relationship between HbA1c, bone microarchitecture/strength and AGEs}

We performed a Pearson's analysis to assess correlations between $\mathrm{HbA} 1 \mathrm{c}$ values collected before surgery and bone parameters obtained by $\mathrm{MCT}$ and biomechanical tests. No significant correlations were found between $\mathrm{HbA} 1 \mathrm{c}$ and measures of bone strength or microarchitecture parameters. Moreover, HbA1c did not correlate with bone AGEs (data not shown).

\section{Discussion}

In the present study, we showed for the first time that T2D patients exhibit lower bone gene expression of bone forming transcriptional factor RUNX2, and upregulated gene expression of the WNT pathway inhibitor SOST. Furthermore, AGEs accumulation was increased in T2D bone compared to non-diabetic bone. Our data strengthen the hypothesis that reduced wone formation in T2D is associated with dysregulated Wnt- $\beta$-catenin signaling pathway, particularly with increases in SOST gene expression. Of note, diabetic patients in our study had well-controlled T2D, suggesting that pathways of bone formation in T2D may be impaired despite good glycemic control.

Previous data have shown that diabetes is characterized by low bone turnover and increased serum sclerostin levels. ${ }^{(7,9,27)}$ For the first time, we provide evidence that, in bones 
from subjects with T2D, the expression of genes involved in the regulation of bone formation is altered. Furthermore, direct analysis of bone specimens showed an increase in bone AGE content.

Accumulation of AGEs in bone might upregulate SOST, which encodes for sclerostin and reduce the expression of $R U N X 2$, a key regulator of bone formation. It is also possible that AGE-associated SOST upregulation mediates transcriptional changes that precede a decrease in bone formation (RUNX2 downregulation), prior to bone density, bone microarchitecture and strength are affected. Our findings here are consistent with prior work that AGEs do not directly impair bone density and strength(25). It is not clear if AGEs accumulation depends on glucose control or disease duration. In this light, osteocytes and sclerostin are likely to play a central role in the pathophysiology of bone fragility in T2D. Osteocytes are the most abundant cell type in the adult skeleton, where they orchestrate bone remodeling by regulating osteoblast and osteoclast activity. ${ }^{(28)}$ It is possible that chronic hyperglycemia and subsequent AGEs accumulation directly affect osteocytes, leading to an increase in sclerostin levels that in turn inhibits bone formation. Importantly, all this process may occur even in well controlled T2D patients, as evident in our subjects. In lact, the majority of our T2D subjects were on monotherapy with oral hypoglycemic medications despite a long disease duration. Preclinical studies showed that both high glucose and AGEs significantly increase the expression of sclerostin protein and mRNA by osteocyte-like MLO-Y4 cells, possibly by increasing TGF- $\beta$ or TNF- $\alpha$, although the underlying mechanisms are still poorly elucidated.(29-31) In clinical studies, circulating sclerostin is almost invariably elevated in hyperglycemic states such as prediabetes, T1D 
and T2D. ${ }^{(32)}$ Furthermore, studies in animal models showed that RUNX2 expression declined after exposure to AGEs, this effect being dependent on sclerostin regulation, as treatment with an anti-sclerostin antibody restored RUNX2 expression. ${ }^{(31)}$ Similarly, a decrease in osteoprogenitor proliferation paralleled a decline in RUNX2 expression in aged ovariectomized rats. RUNX2 gene expression was re-established after the treatment with an anti-sclerostin antibody, indicating sclerostin regulation of RUNX2 gene expression. ${ }^{(33)}$ Finally, our data are in agreement with the observations of Rubin and colleagues, who found that RUNX2 is downregulated in T2D subjects, although this finding was based on analysis of circulating cells expressing bone-related proteins $\left(\mathrm{OCN}^{+} / \mathrm{CD} 146^{+}\right.$cells), and not from the direct analysis of bone tissue. ${ }^{(34)}$ While we found lower RUNX2 expression, ${ }^{(35)}$ osteocalcin gene expression was not significantly different between T2D and non-diabetic subjects. Serum undercarboxylated osteocalcin (Glu-OCN) did not differ between groups, in agreement with gene expression results. Previous studies that assessed circulating osteocalcin in subjects with T2D yielded conflicting findings, with osteocalcin levels either normal or reduced. ${ }^{(32)}$

Measurement of total AGEs in bone showed a significant 1.5 increase in T2D compared to mon-diabetic subjects. This finding is important, because it reveals that even patients with well-controlled T2D present higher levels of AGEs as compared to non-diabetic subjects. AGEs formation and deposition occur over time, and are not affected by short-term changes in glycemic control. Mean duration of disease in T2D subjects was nearly 15 years, therefore it is possible that AGEs deposition in bone had occurred in earlier stages of disease. With respect to total AGEs content in bone, Hunt reported higher levels of pentosidine (a 
biomarker for AGEs cross-links in collagen) and sugar matrix, and no differences in total fluorescent AGEs in T2D bone. ${ }^{(36)}$ Karim showed that total fluorescent AGEs in cortical bone were $\sim 20 \%$ higher in T2D subjects than controls while there were no differences in the trabecula cores. ${ }^{(37)}$ These differences might be due to differences in gender, age of study participants (we enrolled only elderly postmenopausal women, whereas participants in previous studies were younger and mainly male) and, maybe even more relevant, diabetes duration, which was not reported in previous studies but may be an important determinant of total AGEs content.

Our data are in agreement with those from Karim, who found no significant differences in biomechanical properties in bone samples from diabetic versus non-diabetic, except for a trend for lower Young's modulus in the diabetic group. Hunt and colleagues showed a greater tissue mineral content at the femoral neck and increased Young's modulus, yield stress and ultimate stress in T2D subjects versus non-diabetics. ${ }^{(36)}$ Previous clinical studies have shown that T2D may be associated with lower bone strength, as estimated by nanoindentation ${ }^{(38)}$ or $\mathrm{pQCT}{ }^{(39)}$ Differences with our study may be related to the different clinical features of the study population or the use of indirect estimates of bone strength in previous studies, while our observations originate from direct evaluation on the tissue using compressive tests.

Bone density and bone microarchitecture analyses, obtained on bone specimens by DXA and $\mathrm{mCT}$, respectively, showed no differences between T2D subjects versus non-diabetics, confirming previous data in larger human cohorts. $(13,37,40)$ Moreover, our results are in line with data of trabecular microarchitectural properties reported by Karim and 
Hunt. ${ }^{(36,37)}$ Treating bone fragility in diabetics is challenging ${ }^{(41)}$ and our data may reveal SOST as a possible target. Although other anabolic treatments like teriparatide have shown positive effects in subjects with diabetes, ${ }^{(42)}$ more clinical data will be needed to confirm if targeting WNT signaling will improve fracture risk in diabetics.

Our study has some limitations, such as the number of T2D subjects recruited, lack of separation of cortical versus trabecular bone cores. AGEs were available only in a subset of subjects and we could not differentiate between cortical and trabecular bone compartments; cortical porosity could not be quantified. It is possible that with a larger sample size would have found a significant increase in serum sclerostin in diabetics. Although sclerostin may be involved in the pathogenesis of osteoarthritis, ${ }^{(43)}$ it is unlikely that this condition significantly interfered with our findings since all study subjects were affected with osteoarthritis, suggesting that differences in SOST gene expression were linked to diabetes status. More studies using other osteogenic markers will be needed to confirm the role of WNT signaling in bone fragility in diabetics.

A strength of our study is the use of bone specimens from T2D patients, which allowed direct analysis of local gene expression, microarchitectural, compositional and biomechanical iteatures.

In conclusion, our data show that, despite good glycemic control, T2D is associated with alterations in the expression of genes involved in the regulation of bone formation (upregulation of sclerostin and downregulation of RUNX2) that may partly explain the reduced bone formation in T2D. We also found that T2D was associated with increased AGEs accumulation but no changes in BMD, strength or microarchitectural parameters. Our 
data contribute to the understanding of the pathophysiology of diabetes-related bone fragility, but also highlight its complexity. 


\section{References}

1. Unnikrishnan R, Pradeepa R, Joshi SR, Mohan V. Type 2 Diabetes: Demystifying the Global Epidemic. Diabetes. 2017;66(6):1432-42.

2. Napoli N, Chandran M, Pierroz DD, et al. Mechanisms of diabetes mellitus-induced bone fragility. Nat Rev Endocrinol. 2017;13(4):208-19.

3. Napoli N, Schwartz AV, Schafer AL, et al. Vertebral Fracture Risk in Diabetic Elderly Men: The MrOS Study. J Bone Miner Res. 2017.

4. Rubin MR, Patsch JM. Assessment of bone turnover and bone quality in type 2 diabetic bone disease: current concepts and future directions. Bone Res. 2016;4:16001.

5. Conte C, Epstein S, Napoli N. Insulin resistance and bone: a biological partnership. Acta Diabetol. 2018;55(4):305-14.

6. Russo GT, Giandalia A, Romeo EL, et al. Fracture Risk in Type 2 Diabetes: Current Perspectives and Gender Differences. International Journal of Endocrinology. 2016.

7. Hygum K, Starup-Linde J, Harslof T, Vestergaard P, Langdahl BL. MECHANISMS IN ENDOCRINOLOGY: Diabetes mellitus, a state of low bone turnover - a systematic review and meta-analysis. Eur J Endocrinol. 2017;176(3):R137-R57.

8. Starup-Linde J, Lykkeboe S, Gregersen S, et al. Differences in biochemical bone markers by diabetes type and the impact of glucose. Bone. 2016;83:149-55.

9. Starup-Linde J, Vestergaard P. Biochemical bone turnover markers in diabetes mellitus - A systematic review. Bone. 2016;82:69-78.

10. Delgado-Calle J, Sato AY, Bellido T. Role and mechanism of action of sclerostin in bone. Bone. 2017;96:29-37. 
11. Ma YH, Schwartz AV, Sigurdsson S, et al. Circulating sclerostin associated with vertebral bone marrow fat in older men but not women. J Clin Endocrinol Metab. 2014;99(12):E258490.

12. Black DM, Rosen CJ. Clinical Practice. Postmenopausal Osteoporosis. N Engl J Med. 2016;374(3):254-62.

13. Furst JR, Bandeira LC, Fan WW, et al. Advanced Glycation Endproducts and Bone Material Strength in Type 2 Diabetes. J Clin Endocrinol Metab. 2016;101(6):2502-10.

14. Rubin MR. Skeletal fragility in diabetes. Ann N Y Acad Sci. 2017;1402(1):18-30.

15. American Diabetes Association. (2) Classification and diagnosis of diabetes. Diabetes Care. 2015;38 Suppl:S8-S16.

16. Ohman C, Baleani M, Perilli E, et al. Mechanical testing of cancellous bone from the femoral head: experimental errors due to off-axis measurements. J Biomech. 2007;40(11):2426-33.

17. Rio DC, Ares M, Jr., Hannon GJ, Nilsen TW. Purification of RNA using TRIzol (TRI reagent). Cold Spring Harb Protoc. 2010;2010(6):pdb prot5439.

18. Huggett J, Dheda K, Bustin S, Zumla A. Real-time RT-PCR normalisation; strategies and considerations. Genes Immun. 2005;6(4):279-84.

19. Nolan T, Hands RE, Bustin SA. Quantification of mRNA using real-time RT-PCR. Nat Protoc. 2006;1(3):1559-82.

20. Wang Q, Ishikawa T, Michiue T, Zhu BL, Guan DW, Maeda H. Stability of endogenous reference genes in postmortem human brains for normalization of quantitative real-time PCR data: comprehensive evaluation using geNorm, NormFinder, and BestKeeper. Int J Legal Med. 2012;126(6):943-52.

21. Samelson EJ, Demissie S, Cupples LA, et al. Diabetes and Deficits in Cortical Bone Density, Microarchitecture, and Bone Size: Framingham HR-pQCT Study. J Bone Miner Res. 2017. 
22. Ciarelli TE, Fyhrie DP, Schaffler MB, Goldstein SA. Variations in three-dimensional cancellous bone architecture of the proximal femur in female hip fractures and in controls. $\mathrm{J}$ Bone Miner Res. 2000;15(1):32-40.

23. Wolfram U, Schwiedrzik J. Post-yield and failure properties of cortical bone. Bonekey Rep. 2016;5:829.

24. Abraham AC, Agarwalla A, Yadavalli A, McAndrew C, Liu JY, Tang SY. Multiscale Predictors of Femoral Neck In Situ Strength in Aging Women: Contributions of BMD, Cortical Porosity, Reference Point Indentation, and Nonenzymatic Glycation. J Bone Miner Res. 2015;30(12):2207-14.

25. Tang SY, Zeenath U, Vashishth D. Effects of non-enzymatic glycation on cancellous bone fragility. Bone. 2007;40(4):1144-51.

26. World Health Organization. WHO Scientific Group on the Assessment of Osteoporosis at Primary Health Care Level. Summary Meeting Report. Available at https://www.who.int/chp/topics/Osteoporosis.pdf; Last accessed on April 12, 2020. 2007.

27. Starup-Linde J, Eriksen SA, Lykkeboe S, Handberg A, Vestergaard P. Biochemical markers of bone turnover in diabetes patients--a meta-analysis, and a methodological study on the effects of glucose on bone markers. Osteoporos Int. 2014;25(6):1697-708.

28. Boyce BF, Yao Z, Xing L. Osteoclasts have multiple roles in bone in addition to bone resorption. Crit Rev Eukaryot Gene Expr. 2009;19(3):171-80.

29. Miranda C, Giner M, Montoya MJ, Vazquez MA, Miranda MJ, Perez-Cano R. Influence of high glucose and advanced glycation end-products (ages) levels in human osteoblast-like cells gene expression. BMC Musculoskelet Disord. 2016;17:377. 
30. Notsu M, Kanazawa I, Takeno A, et al. Advanced Glycation End Product 3 (AGE3) Increases Apoptosis and the Expression of Sclerostin by Stimulating TGF-beta Expression and Secretion in Osteocyte-Like MLO-Y4-A2 Cells. Calcif Tissue Int. 2017;100(4):402-11.

31. Sakamoto E, Kido JI, Takagi R, et al. Advanced glycation end-product 2 and Porphyromonas gingivalis lipopolysaccharide increase sclerostin expression in mouse osteocyte-like cells. Bone. 2019;122:22-30.

32. Costantini S, Conte C. Bone health in diabetes and prediabetes. World J Diabetes. 2019;10(8):421-45.

33. Boyce RW, Brown D, Felx M, et al. Decreased osteoprogenitor proliferation precedes attenuation of cancellous bone formation in ovariectomized rats treated with sclerostin antibody. Bone Rep. 2018;8:90-4.

34. Manavalan JS, Cremers S, Dempster DW, et al. Circulating osteogenic precursor cells in type 2 diabetes mellitus. The Journal of Clinical Endocrinology and Metabolism. 2012;97(9):3240-50.

35. Atashi F, Modarressi A, Pepper MS. The role of reactive oxygen species in mesenchymal stem cell adipogenic and osteogenic differentiation: a review. Stem Cells Dev. 2015;24(10):1150-63.

36. Hunt $\mathrm{H}$, Torres $\mathrm{A}$, Palomino $\mathrm{P}$, et al. Altered tissue composition, microarchitecture, and mechanical performance in cancellous bone from men with type 2 diabetes mellitus. $\mathrm{J}$ Bone Miner Res. 2019.

37. Karim L, Moulton J, Van Vliet M, et al. Bone microarchitecture, biomechanical properties, and advanced glycation end-products in the proximal femur of adults with type 2 diabetes. Bone. 2018;114:32-9. 
38. Farr JN, Khosla S. Determinants of bone strength and quality in diabetes mellitus in humans. Bone. 2016;82:28-34.

39. Petit MA, Paudel ML, Taylor BC, et al. Bone mass and strength in older men with type 2 diabetes: the Osteoporotic Fractures in Men Study. J Bone Miner Res. 2010;25(2):285-91.

40. Samelson EJ, Demissie S, Cupples LA, et al. Diabetes and Deficits in Cortical Bone Density, Microarchitecture, and Bone Size: Framingham HR-pQCT Study. J Bone Miner Res. 2018;33(1):54-62.

41. Ferrari SL, Abrahamsen B, Napoli N, et al. Diagnosis and management of bone fragility in diabetes: an emerging challenge. Osteoporos Int. 2018;29(12):2585-96.

42. Langdahl BL, Silverman S, Fujiwara S, et al. Real-world effectiveness of teriparatide on fracture reduction in patients with osteoporosis and comorbidities or risk factors for fractures: Integrated analysis of 4 prospective observational studies. Bone. 2018;116:58-66.

43. Weivoda MM, Youssef SJ, Oursler MJ. Sclerostin expression and functions beyond the osteocyte. Bone. 2017;96:45-50.

\section{Acknowledgements}

The study was supported by a Campus Bio-Medico University strategic grant .

\section{Authors' roles}

Study design: NN, RS, PP. Data collection: AP, FC, FR, VG, Cl, GL, CCQ, CM, GV, SJT. Data analysis: CP, SJT, SS. Data interpretation: NN, AP, RS, SJT, MJS, RP, SS, CC, TB, 
MM, VD. Drafting manuscript: NN, AP, RS, GL, CC. Approving final version of manuscript: ALL. NN, CP, RS and AP take responsibility for the integrity of the data analysis 
Tables

Table 1.

\begin{tabular}{lccc}
\hline & $\begin{array}{c}\text { Non-diabetic } \\
\text { subjects }\end{array}$ & $\begin{array}{c}\text { T2D subjects } \\
(\mathbf{n = 1 9 )}\end{array}$ & value \\
& $(\mathbf{n = 7 3 )}$ & & \\
\hline Age (years) & $73.2(5.8)$ & $75.2(8.5)$ & NS \\
BMI (kg/m $\left.{ }^{2}\right)$ & $27.7(5.6)$ & $29.9(5.4)$ & NS \\
Menopausal age (years) & $49.3(5.2)$ & $48.9(5.9)$ & NS \\
Pregnancies (n) & $2.0(1.2)$ & $2.5(1.3)$ & NS \\
Disease duration (years) & - & $14.6(9.8)$ & - \\
Fasting plasma glucose (mg/dl) & $100.7(11.21)$ & $120.1(21.5)$ & 0.002 \\
HbA1c (\%) & - & $6.5(1.7)$ & - \\
Serum calcium (mg/dl) & $9.2(0.4)$ & $9.3(0.5)$ & NS \\
Creatinine (mg/dl) & $0.76(0.16)$ & $0.83(0.25)$ & NS \\
Serum blood urea nitrogen (mg/dl) & $41.5(11.5)$ & $48 .(15.6)$ & NS \\
\hline
\end{tabular}

Table 1. Demographic and clinical features of the study subjects. Results are presented as mean (SD). 
Table 2.

Non-diabetic

T2D subjects

$P$ value

subjects

\section{Dual-energy X-ray absorptiometry}

\begin{tabular}{llcc}
\hline BMD Lumbar L1+L4 (g/cm²) & $0.92(0.17)$ & $0.95(0.12)$ & 0.43 \\
Lumbar T-score (SD) & $-1.15(1.43)$ & $-0.83(1.14)$ & 0.44 \\
BMD Femoral total $\left(\mathrm{g} / \mathrm{cm}^{2}\right)$ & $0.79(0.11)$ & $0.82(0.09)$ & 0.39 \\
BMD Femoral neck (g/cm²) & $0.68(0.12)$ & $0.66(0.11)$ & 0.62 \\
Femoral T-score (SD) & $-1.36(0.87)$ & $-0.98(0.75)$ & 0.27
\end{tabular}

\section{Microcomputed Tomography}

\begin{tabular}{lccc}
\hline BV/TV & $0.26(0.10)$ & $0.24(0.14)$ & 0.54 \\
Connectivity $\left(1 / \mathrm{mm}^{3}\right)$ & $6.75(2.41)$ & $6.99(4.25)$ & 0.89 \\
Trabecular number $(1 / \mathrm{mm})$ & $1.59(0.37)$ & $1.48(0.5)$ & 0.49 \\
Trabecular Thickness (mm) & $0.2(0.08)$ & $0.2(0.05)$ & 0.98 \\
Trabecular Separation (mm) & $0.61(0.15)$ & $0.71(0.2)$ & 0.14 \\
Volumetric BMD (mgHA/cm $\left.{ }^{3}\right)$ & $243.0(74.8)$ & $220.4(100.2)$ & 0.48 \\
Tissue mineral density (mgHA/cm $\left.{ }^{3}\right)$ & $685.5(29.51)$ & $689.36(32.8)$ & 0.65 \\
\hline Biomechanical Properties & & & 0.4 \\
\hline Young's modulus (MPa) & $58.93(52.39)$ & $43.16(55.91)$ & 0.97 \\
Ultimate strength (MPa) & $5.38(3.12)$ & $8.17(10.51)$ & 0.13 \\
Yield strength (MPa) & $4.87(2.64)$ & $3.78(3.40)$ & \\
\hline
\end{tabular}


Table 2. Bone density, bone microarchitecture and biomechanical parameters in nondiabetic and T2D subjects. Data are presented as Mean (SD).

\section{Figure legends}

Figure 1. Gene expression analyses in bone samples. Data are expressed as logarithmic scale medians and interquartile ranges for non-diabetics and T2D subjects. A. SOST mRNA levels resulted higher in T2D subjects vs. non-diabetics $(p=0.006$, Figure 1.A), even independently of age and BMI $(p=0.007)$. B. RUNX2 gene expression was lower in T2D subjects vs. non-diabetics $(p=0.025)$. C.OCN levels were not different between groups $(p=0.72)$.

Figure 2. AGEs content in bone samples of non-diabetic (dark grey bar) and T2D subjects (light grey bar). Data are expressed as Mean \pm SEM. T2D $(n=5)$ had higher AGEs bone content compared to non-diabetics $(n=15)(364.8 \pm 78.2 \mu$ g quinine/g collagen vs. $209.9 \pm$ 34.4 $\mu$ g quinine/g collagen, respectively, $p<0.001)$.

Figure 3. Relationship between AGEs content and bone microarchitecture in T2D and nondiabetic subjects: AGEs were negatively correlated with volumetric BMD (A), BV/TV (B) and positively correlated with trabecular separation and spacing (C). 
Figure 1

A

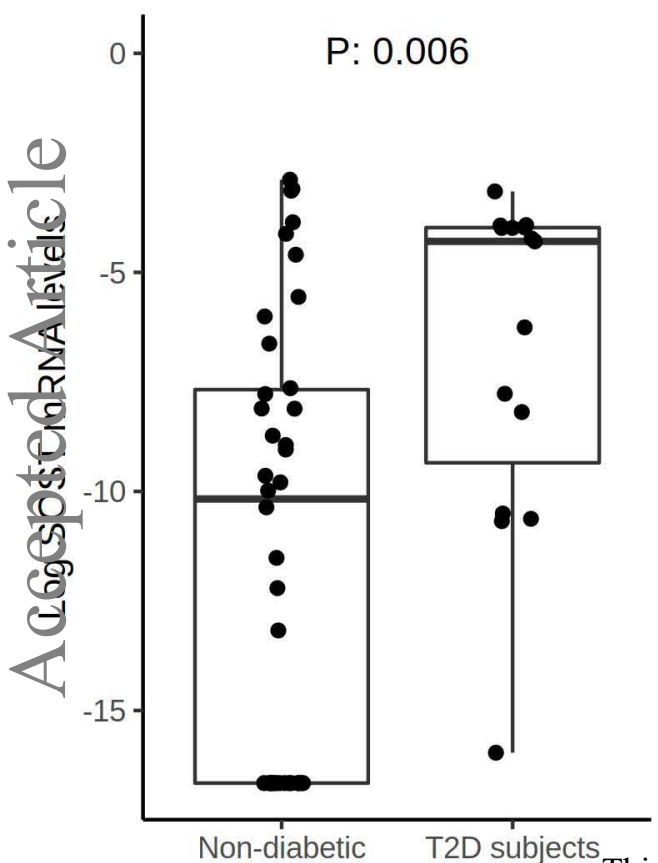

Non-diabetic subjects
B

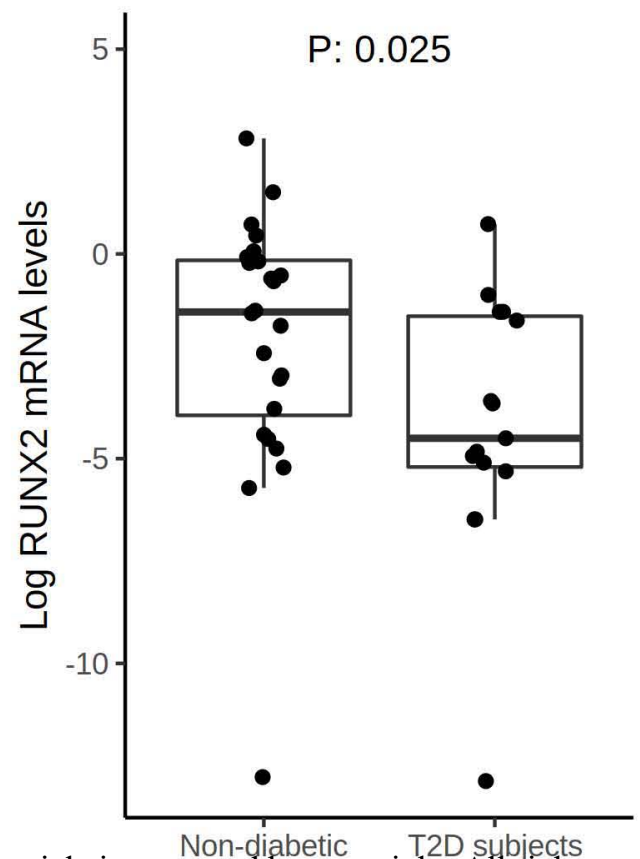

This article is protectedjjeytscopyright. Afl rights reserved.
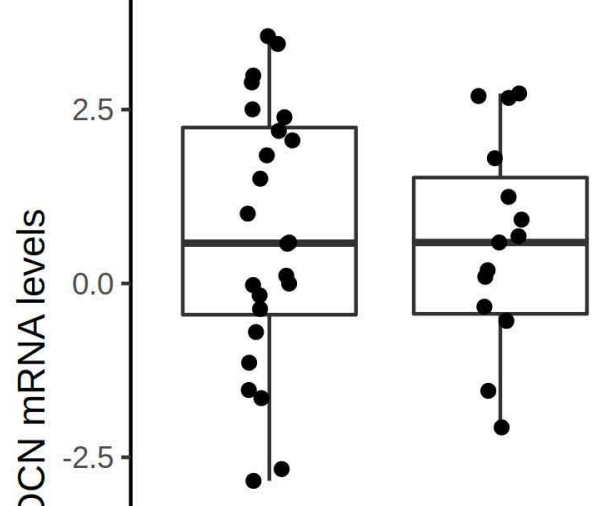

$P: 0.721$ 
Figure 2

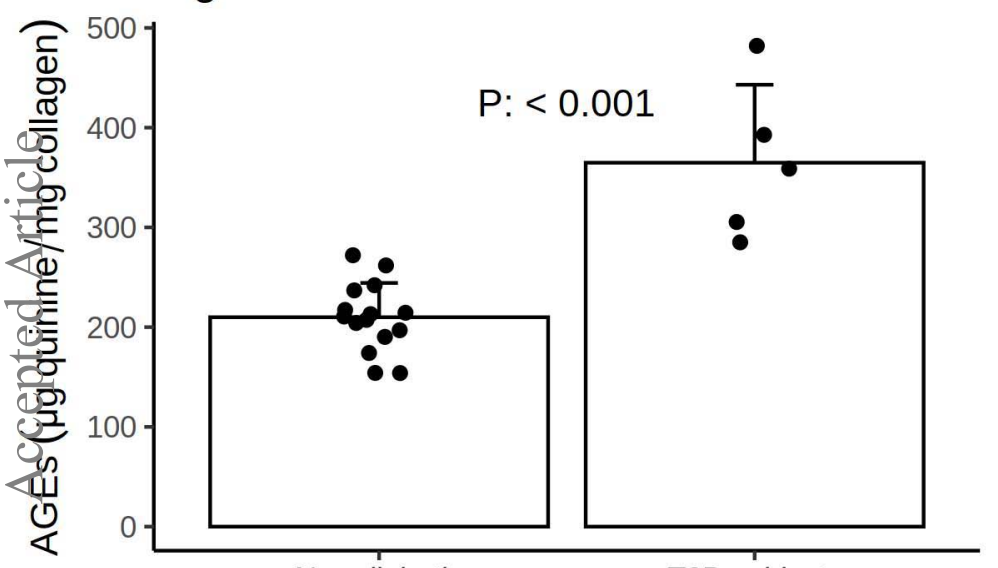

This article is plootedieded by copyright. AlTaghas piesteved. subjects 
Figure 3
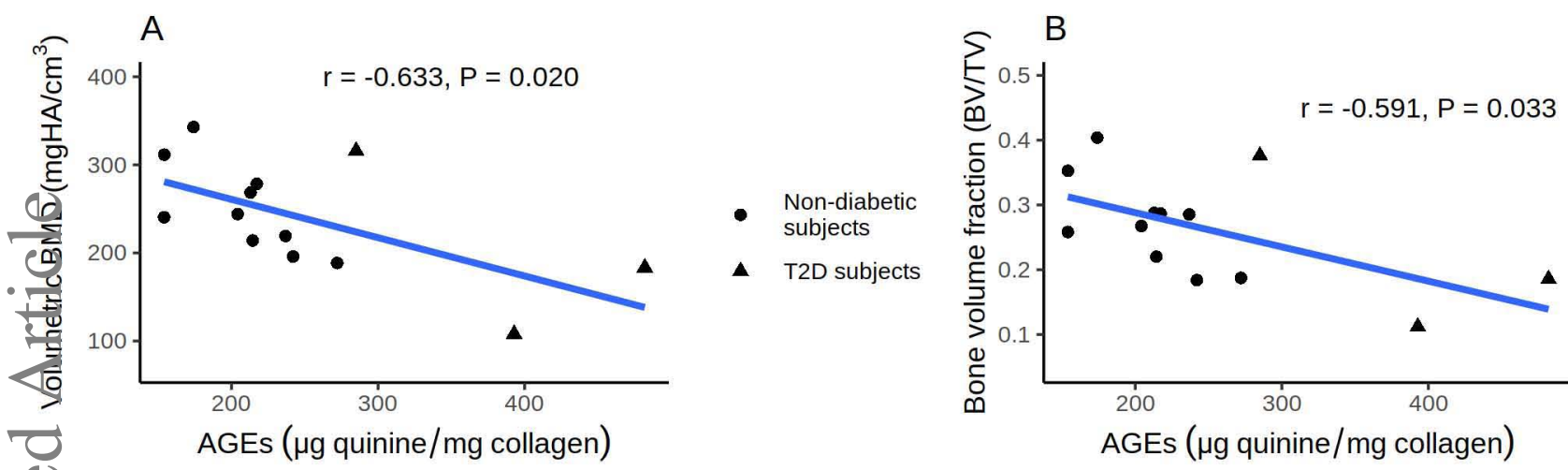

Non-diabetic

Non-diabetic subjects

- T2D subjects

C

$$
r=0.624, P=0.023
$$

$1.00-$

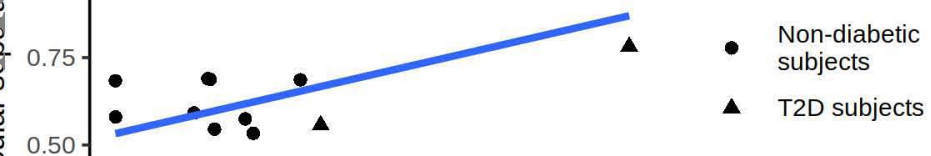

$\bullet$

$200 \quad 300$

400 This article is protected by copyright. All rights reserved.

AGEs ( $\mu$ g quinine/mg collagen) 